\title{
Correction to: eDNA detection of corallivorous seastar (Acanthaster cf. solaris) outbreaks on the Great Barrier Reef using digital droplet PCR
}

\author{
Sven Uthicke ${ }^{1}$ (I) Miles Lamare $^{2} \cdot$ Jason R. Doyle $^{1}$
}

Published online: 18 August 2021

(C) Springer-Verlag GmbH Germany, part of Springer Nature 2021

\section{Correction to: Coral Reefs (2018) 37:1229-1239 https://doi.org/10.1007/s00338-018- 1734-6}

In the published paper are minor discrepancies between the supplementary table and the data we show in the main text (Table 2, Fig. 3). Due to a change of analytical methods, we had achieved a lower Limit of Quantification (LOQ) for the final data, but presented the initial data in the supplementary material. In addition, an error occurred when averaging some of the datapoints. Here, we present and updated version of Table 2 (statistical analyses) and Fig. 3 (graphical presentation of the linear model results). Changes to data, statistics and graphs are only minor and have no consequence to the conclusions of our publication.
Table 2 Results of linear models comparing eDNA concentrations and the percentage of samples above the Limit of Quantification (LOQ) collected at depth (underwater) or on the ocean surface (surface), and densities of Acanthaster cf. solaris (CoTS density) (Updated Table 2)

\begin{tabular}{lccccc}
\hline Comparison & Estimate & SE & $t$ & $p$ & Model $R^{2}$ \\
\hline Under water concentration versus CoTS density & \\
Intercept & 2.5794 & 0.1164 & 22.16 & $<0.0001$ & 0.63 \\
Slope & 0.9805 & 0.1640 & 5.98 & $<0.0001$ & \\
Surface concentration versus CoTS density & & \\
Intercept & 2.3157 & 0.1399 & 16.55 & $<0.0001$ & 0.43 \\
Slope & 0.7572 & 0.1933 & 3.92 & 0.0010 & \\
Under water $\%>$ LOQ versus CoTS density & & \\
Intercept & 1.3275 & 0.0811 & 16.37 & $<0.0001$ & 0.67 \\
Slope & 0.6792 & 0.1097 & 6.19 & $<0.0001$ & \\
Surface \% > LOQ versus CoTS density & & \\
Intercept & 1.5790 & 0.0639 & 24.70 & $<0.0001$ & 0.49 \\
Slope & 0.3878 & 0.0883 & 4.39 & 0.0004 & \\
\hline
\end{tabular}

The original article can be found online at https://doi.org/10.1007/ s00338-018-1734-6.

\section{Sven Uthicke}

suthicke@aims.gov.au

1 Australian Institute of Marine Science, PMB No. 3, Townsville MC, QLD 4810, Australia

2 Department of Marine Science, University of Otago, Dunedin 9016, New Zealand 
Under Water
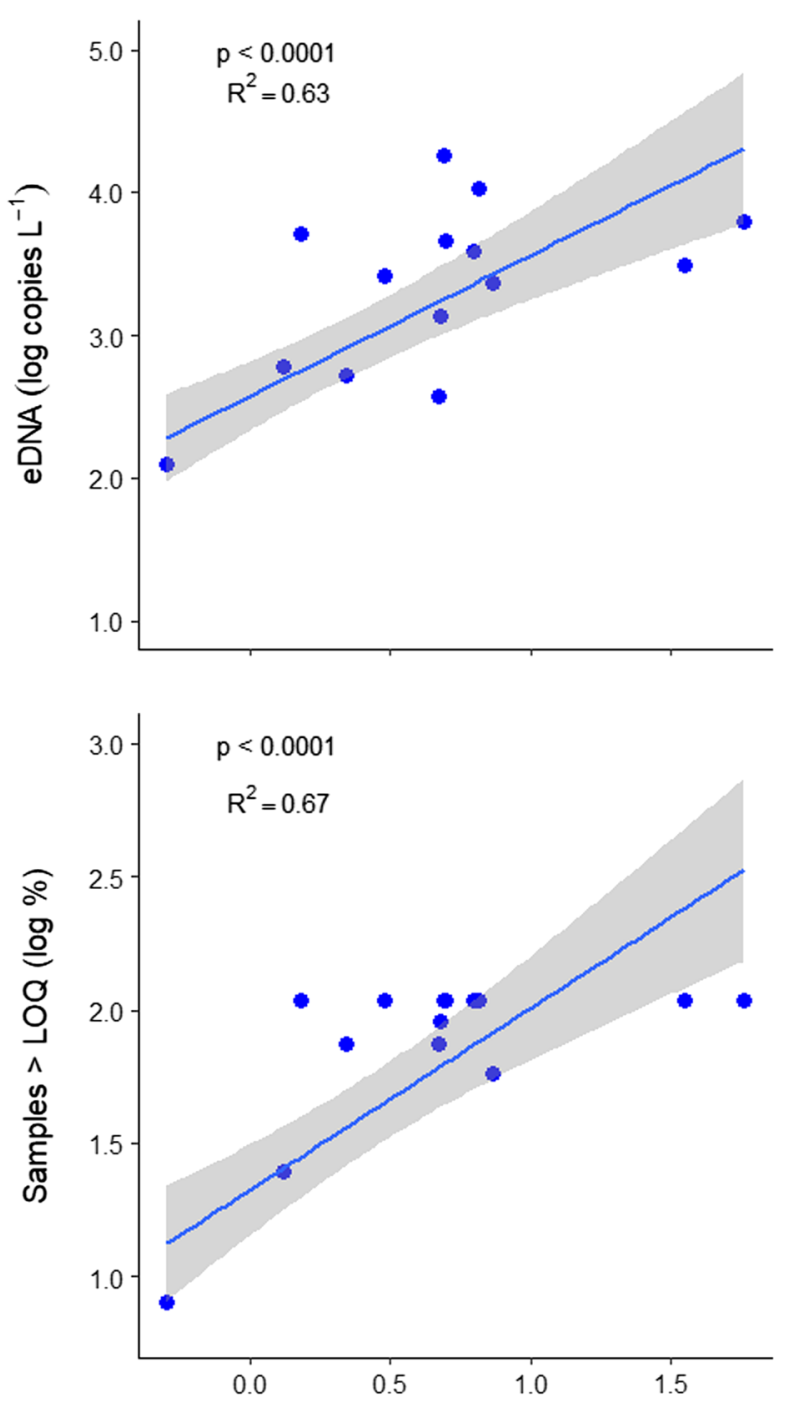

\section{Surface}

$p=0.0010$

$\mathrm{R}^{2}=0.43$

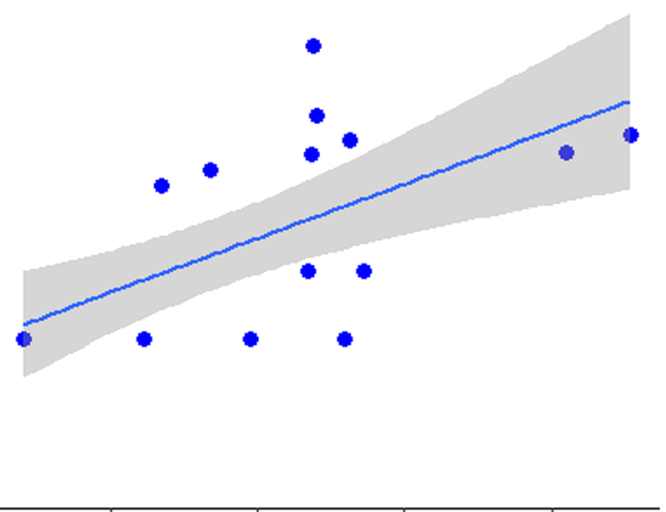

$p=0.0004$

$R^{2}=0.49$
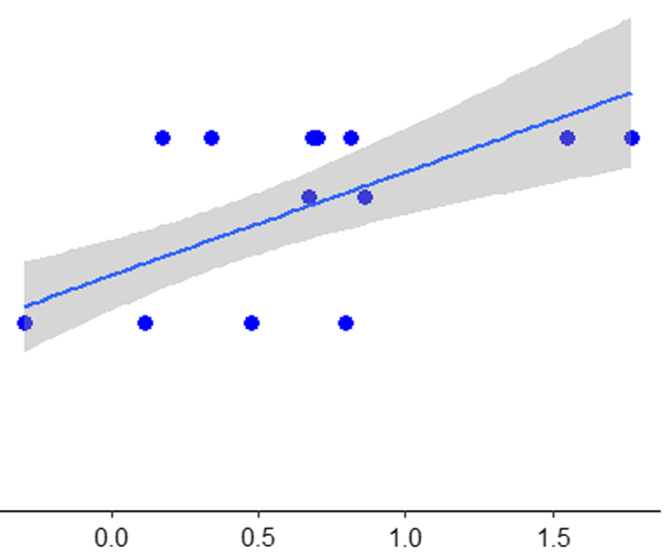

Acanthaster cf. solaris density (log Ind. $100 \mathrm{~m}^{-2}$ )

Fig. 3 Linear models of eDNA in water samples taken in situ (underwater) and on the sea surface (surface) as a function of Acanthaster cf. solaris densities on reefs of the Great Barrier Reef, Australia. Response variables are either the average concentration per site (eDNA) or the proportion of samples above the Limit of Quantification (LOQ). The grey shaded area represents 1 SE of each fit. Regression equations (intercepts and slopes) for the models are given in Table 2. All variables were $\log 10(9)$ transformed prior to analyses. For transformation, 0.59 the minimum value observed for the respective variable was added to all values to accommodate zero values (Updated Fig. 3)

Publisher's Note Springer Nature remains neutral with regard to jurisdictional claims in published maps and institutional affiliations. 